\title{
GEEC: Graphic editor of electrical circuits
}

\author{
Filip Paulů, Jiří Hospodka \\ Department of Circuit Theory \\ Czech Technical University in Prague \\ Prague, Czech Republic \\ Email: paulufil@fel.cvut.cz, hospodka@fel.cvut.cz
}

\begin{abstract}
This paper presents web-based graphical schematic editor for analysis of electric and electronic circuits. It is a JavaScript application that uses Spice OPUS and Maple with PraCAn package as a computation engine. In case of linear circuits, results can be obtained in symbolic form. Continuous-time linear and nonlinear circuits as well as periodically switched linear (PSL) circuits can be analyzed. All created circuits can be exported to various file formats for greater compatibility with documentation formats and other simulation programs. The whole system is developed at the Department of Circuit Theory, for teaching support and research.

Index Terms-graphic editor of electrical circuits; webbased application; analysis; electric circuits;
\end{abstract}

\section{INTRODUCTION}

Web-applications have a plenty of advantages against the common desktop ones. One of the greatest advantages is the possibility of working from anywhere where there is an internet connection. Another advantage is that there is no installation required which also means that there's no platform dependency. Plus, applications that allow us to save our work on a server ensures we can work from anywhere without a need to use any external portable or cloud storages. All of these things above make the application not only simpler to use and grasp for students but also make the work easier for research workers [1] [12] [13]. Some good examples of such applications are web-based electrical circuit simulators (EVCLab) [10] or webbased application specialized in Standard Cell Circuits [9].

Nowadays, plenty of programs specializes in electrical circuit analysis. Most of these systems uses programs such as SPICE [4] to perform the analysis, e.g., Micro-Cap [6], TINA, PSpice [5], CircuitLab, Win-Spice [7]. Many of those give only numerical results. Hardly ever we can find programs that would give us the results in symbolic or semi-symbolic form [8] [3] and analysis of periodically switched linear (PSL) circuits, i.e., a circuit with switched capacitors (SC) or switched currents (SI) [2].

Electrical circuits simulators use research workres from various scientific fields e.g., scientits who study nonlinear finance system with the use of Cadence OrCAD [14]. On the other hand, there are also students, who have never used similar programs before. For that reason, it is necessary to make the applications user-friendly and self-explanatory even for the first time users with no more than basic electrotechnical background. At the same time, it mustn't lack any functionality necessary for everyday work.

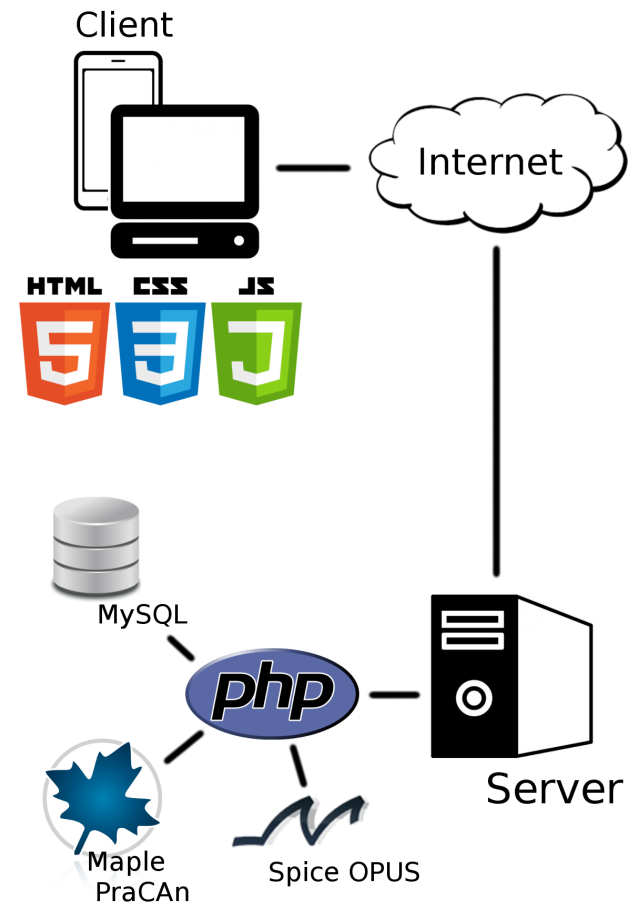

Fig. 2: Client-server concept

\section{WEB-BASED APPLICATION}

The application presented in this article (GEEC Fig. 1) helps users to create an schematic circuit and analyze it in many different ways. The circuit can be saved as a file of various formats for greater compatibility with other programs. Whole application aims to be self-explanatory so no manual is needed.

\section{A. Implementation}

This application is based on client-server concept (Fig. 2), which uses a special simulating programs on the server side. That way, the user can use this application anywhere, anytime as long as he or she knows application's URL address and has an internet connection access.

There was a possibility to use Java or Flash for the client side. These technologies require an installation of plugins. On the other hand, JavaScript programs only need any modern browser (e.g. Mozilla Firefox, Goggle Chrome, Opera, etc.) that is usually already installed on any commonly used computer. JavaScript is natively supported in these browsers, so it doesn't need anything else to work. Therefore this program has been developing as JavaScript program with the 


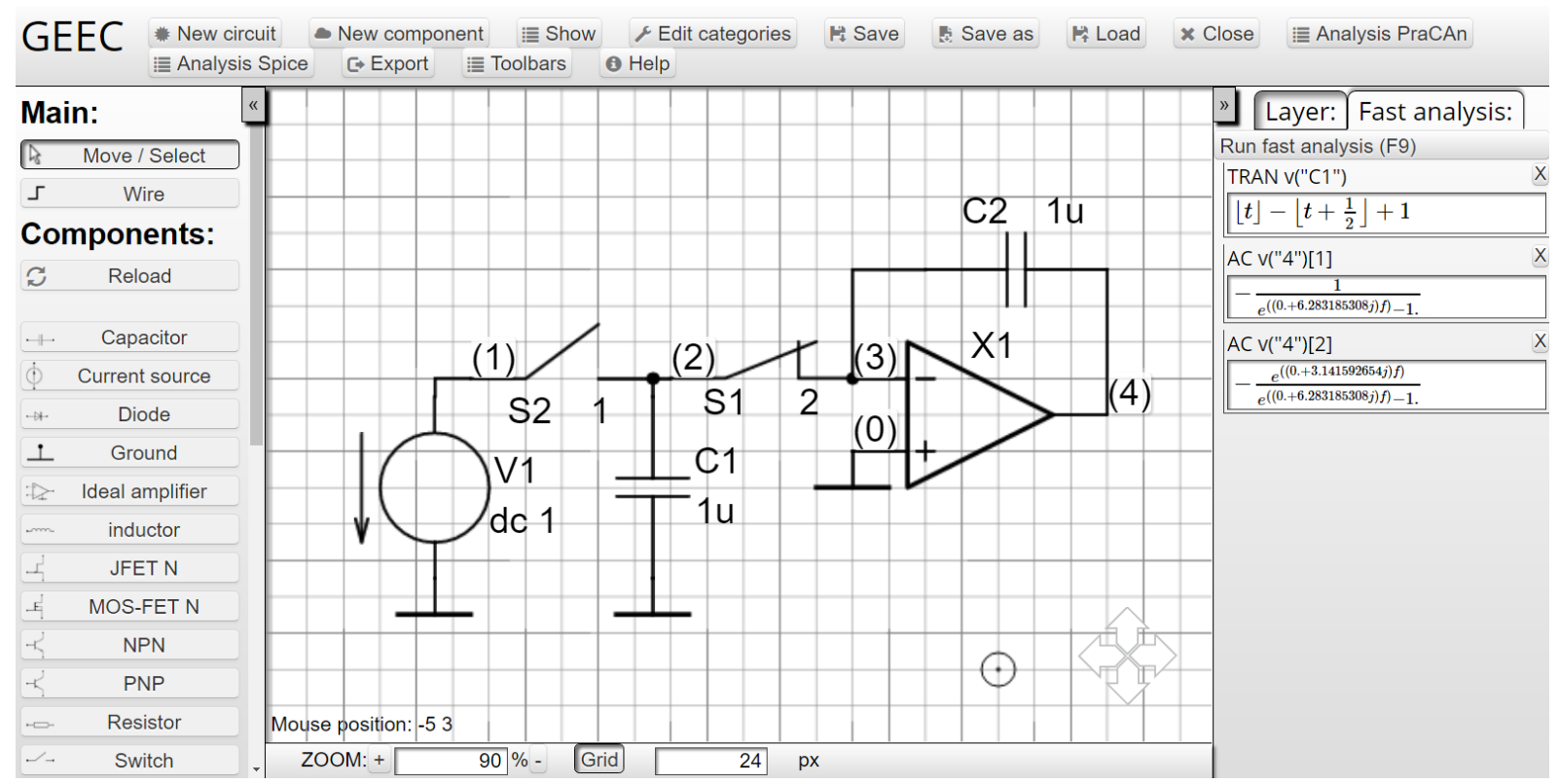

Fig. 1: Graphic editor of electrical circuits (GEEC)

help of ECMA Script 6 syntax [16]. This program uses fat client technology to keep most of the application logic on the client side. The client communicates with the server only when data, such as analysis results, are transferred via AJAX technology [17] using JSON syntax. In this way, demands for the quality of internet connection are kept low.

Server side serves only as a data storage and a middleman between the client side and programs for electrical circuit analysis. That reduces server requirements to handling database and running programs for analysis. These requirements are met by nearly all available technologies. To keep things simple, this program uses PHP with MySQL database.

From the perspective of a user, the application composes out of three parts: Graphics editor, electronic component editor and schematic editor which is bound to analysis programs.

\section{B. Electronic components}

It is necessary for users to be able to change some components' parameters or even omit them completely. On the other hand, not all parameters can be changed or left out, because it could interfere with the application's compatibility with programs that run an analysis. To ensure this compatibility, all created electric circuits can be exported into commonly used NetList file. That means for each component to have its own NetList record to make export possible. To remove the necessity of NetList syntax knowledge for basic users, it is left to administrators of the application to create new electronic components and to set which parameters can be changed and how. Afterward, the administrator needs to define its interpretation to NetList syntax. As an example, you can see editing of linear OpAmp parameters on Fig. 3.

Program administrators use GEEC's own graphical editor, that is available to all users, to create new components and to edit the current ones. Editor's tools

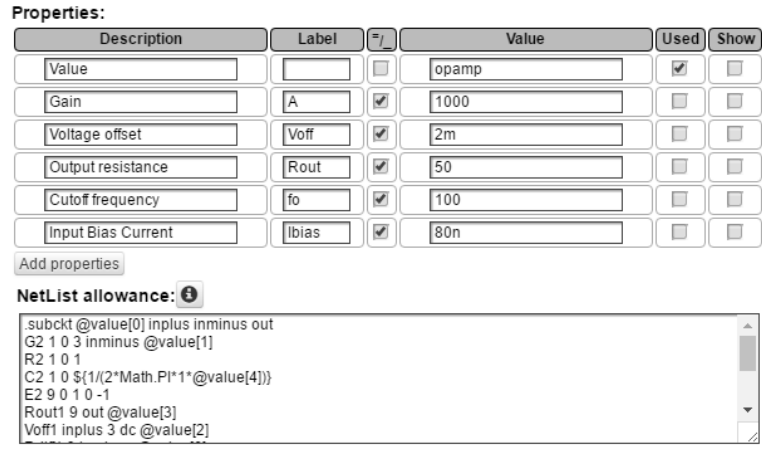

Fig. 3: Editing OpAmps parametres

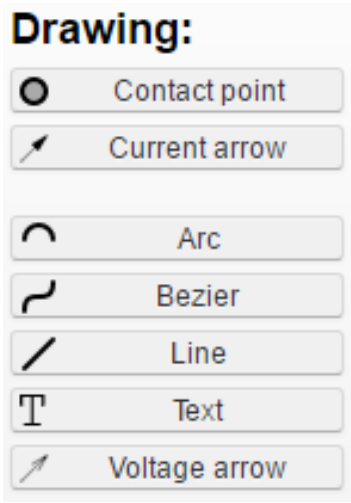

(a) Drawing tools

\begin{tabular}{|c|c|}
\hline L Layer: $\mathrm{F}$ & Fast analysis: \\
\hline \multicolumn{2}{|c|}{ Run fast analysis (F9) } \\
\hline$A C$ v("C1") & $x$ \\
\hline$\frac{\mathrm{V} 1}{2 j \mathrm{C} 1 \pi f \mathrm{R} 1+1}$ & \\
\hline TRAN v("C1") & $x$ \\
\hline \multicolumn{2}{|c|}{$10-10 e^{(-100 t)}$} \\
\hline $\begin{array}{l}\text { TRAN v("C1") } \\
t=0.01\end{array}$ & $\underline{x}$ \\
\hline 6.321205588 & \\
\hline TF v("C1") & $\underline{x}$ \\
\hline \begin{tabular}{|c|} 
V1 \\
$\mathrm{C} 1 s \mathrm{R} 1+1$ \\
\end{tabular} & \\
\hline TF v("C1") & $\underline{x}$ \\
\hline$\frac{100}{s+100}$ & \\
\hline
\end{tabular}

(b) Fast Analysis panel
Fig. 4: Panels

can be seen on Fig. 4 (a). Certain styles (Fig. 5) can be applied to any tool.

Component catalog in similar programs is usually included by additional libraries. The GEEC allows the administrators to connect each component separately to any component catalog that has access to the database. At the moment, the program uses the catalog 


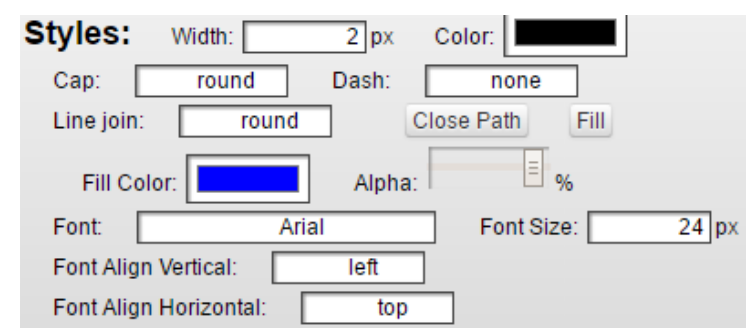

Fig. 5: Styles for drawing tools

TABLE I: OP analysis of voltage divider with use of PraCAn package

\begin{tabular}{c|c|c} 
Name & Symbolic & Numerical \\
\hline $\mathrm{i}(" \mathrm{R} 1 ")$ & $\frac{V 1(R 2+R 3)}{R 1 R 2+R 1 R 3+R 2 R 3}$ & $\frac{1}{1500}$ \\
$\mathrm{i}(" \mathrm{R} 2 ")$ & $\frac{1}{R 1 R 2+R 1 R 3}$ & $\frac{1}{3000}$ \\
$\mathrm{i}(" \mathrm{R} 3 ")$ & $\frac{R 2 V 1}{R 1 R 2+R 1 R 3+R 2 R 3}$ & $\frac{1}{3000}$ \\
$\mathrm{i}(" \mathrm{~V} 1 ")$ & $-\frac{V 1(R 2+R 3)}{R 1 R 2+R 1 R 3+R 2 R 3}$ & $\frac{-1}{1500}$ \\
$\mathrm{v}(" 1 ")$ & $V 1$ & 10 \\
$\mathrm{v}(" 2 ")$ & $\frac{R 2 R 3 V 1}{R 1 R 2+R 1 R 3+R 2 R 3}$ & $\frac{10}{3}$ \\
$\mathrm{v}(" \mathrm{R} 1 ")$ & $\frac{R 1 V 1(R 2+R 3)}{R 1 R 2+R 1 R 3+R 2 R 3}$ & $\frac{20}{3}$ \\
$\mathrm{v}(" \mathrm{R} 2 ")$ & $\frac{R 2 R 3 V 1}{R 1 R 2+R 1 R 3+R 2 R 3}$ & $\frac{10}{3}$ \\
$\mathrm{v}(" \mathrm{R} 3 ")$ & $\frac{R 2 R 3 V 1}{R 1 R 2+R 1 R 3+R 2 R 3}$ & $\frac{10}{3}$ \\
$\mathrm{v}(" \mathrm{~V} 1 ")$ & $V 1$ & 10
\end{tabular}

at https://asinus.feld.cvut.cz/katalog/.

\section{Electric circuit analysis}

This application uses server-side programs to perform an analysis. The client sends a request for analysis altogether with a created circuit and server forwards it to relevant analysis program. The request is then processed and the result is returned to the user.

GEEC uses two different computation engines to perform analysis to cover as many types of analysis as possible.

The numerical type of analysis is performed by Spice OPUS simulator [11]. The simulator includes an interpreted programming language called Nutmeg, which allows interactive Spice sessions. One of the important reasons to use this simulator is that the Copyright holders grant user the right to use this program free of charge for any purpose and therefore the GEEC as well can be free of charge to use for numerical analysis.

For a symbolic and semi-symbolic type of analysis, PraCAn package is used [15]. PraCAn package is a library of functions for Maple, which facilitates the symbolic and semi-symbolic analysis of continuous and discrete-time linearized circuits. PraCAn is an acronym for Prague Circuits Analyzer. The input syntax for circuit description is nearly the same as in Spice program. The package includes analysis of idealized SC and SI circuits and PSL circuits simulation (switched circuits with nonideal effects) [3].

GEEC can perform OP (Operation Point) analysis and give both, numerical and symbolic results (find voltage divider example in table I).

GEEC can perform common DC, AC and Transient analysis that give results in an interactive chart in

\section{AC analysis}
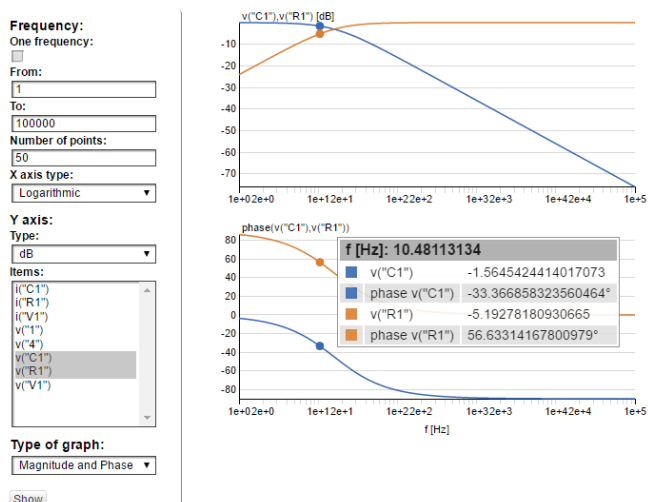

Fig. 6: AC low-pass filter analysis

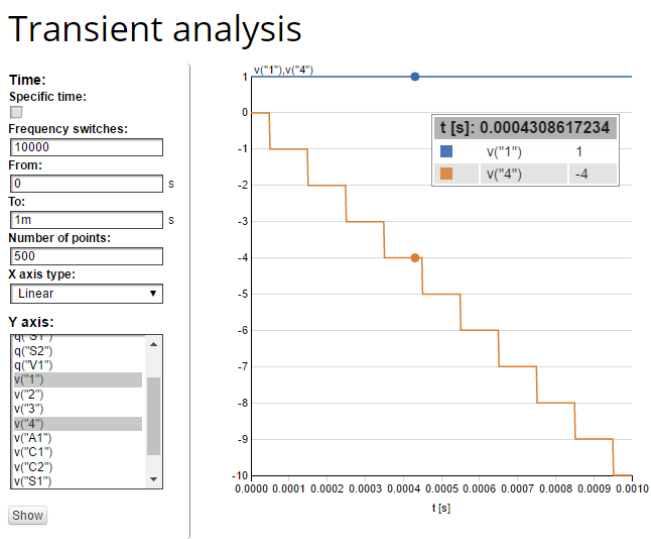

Fig. 7: PSL circuit analysis

Fig. 6. These analyses can be done through both Spic OPUS or PraCAn.

GEEC can also perform less common AC, DC, Transient and Transfer Function analysis with use of PraCAn packgage which give numerical and symbolic results. As an example see TF symbolic result (1) and AC numerical result (2) of low-pass filter analysis.

$$
\begin{gathered}
V_{C_{1}}=\frac{V_{1}}{2 j C_{1} \pi f R_{1}+1} \\
V_{C_{1}}=\frac{100}{(0+6.283185308 j) f+100}
\end{gathered}
$$

GEEC can as well analyze periodically switched linear (PSL) circuit. See example of PSL circuit (Fig. 1) and a result in a chart of transient analysis (Fig. 7).

The application also allows you to get multiple results of circuit analysis at once. On the right panel, there is a Fast Analysis section where user can select which analysis result you want to display. When the circuit is modified, the Fast Analysis section can show the user all changes in results right away, so the user can keep a clear view of all important properties of the circuit (see the fast analysis on Fig. 4 (b).

\section{Export}

GEEC allows you to export electric circuit in a format that is suitable for other programs, which allows 
the user to further use the created circuit in other files or to analyze it in different ways than GEEC provides.

Pixel formats, i.e., PNG and JPG format, are particularly useful when creating examples or presentations for students. For use in articles or printed lecture notes, it is better to use vector formats of which GEEC offers EPS and PDF format.

It is also possible to export circuit in a way that is suitable for $\mathrm{IT}_{\mathrm{E} X \mathrm{X}}$ files. This export gives you two files. One with pictures in EPS or PDF format, and other with text in format for LTEXsyntax. This way, it is still possible to further edit and stylize the text in the LTEXdocument.

For further research and analysis of electrical circuits created in GEEC, NetList format, which is commonly used in other simulation programs, is also available. A circuit can also be exported to Maple (PraCAn package) format on which symbolic calculations can be done.

\section{E. User interface}

The user interface of GEEC is focused not only on users who use similar programs on a daily basis but also on users who have very little or none experience with similar programs. That means that the program's user interface should offer enough possibilities and routine operation options for the experienced users but should not be too overwhelming and confusing to beginners. The balance between those two views is quite difficult to reach.

This application has been tested on participants from both these groups and their feedback was used to improve the user interface of the application into the current state (Fig. 1).

Thanks to this modifications, the application became more intuitive and more users started to use it.

\section{CONCLUSIONS}

The web-based graphic editor of electrical circuits has been created to enable analysis of electric and electronic circuits for a wide range of users. Designed system combines technologies as fat client technology, JavaScript, PHP and circuit simulation, and provides the user with vivid interface, convenient operation and powerful simulation capability. The application uses facilities of Spice OPUS program for numeric analysis and PraCAn package in Maple program for symbolic and semi-symbolic analysis of continuous-time and periodically switched linear circuits. Any known webbased system doesn't contain such capabilities.

This application was created on Faculty of Electrical Engineering, Czech Technical University (CTU) in Prague for teaching support and research. The usage of the application is easy and intuitive. It doesn't require any program installation and can be used even without any command syntax knowledge.

This program has already come into use in education and research. Thanks to its intuitive controls, it helps students to learn electric circuits and electronics. Scientists use this program for electric circuits analyses. It is also often used for its ability to export circuits to graphical formats, which can be widely used in presentations and articles.

The system is open to all users from CTU domain https://asinus.feld.cvut.cz/geec although analyzis using PraCAn package need Maple program that is restricted by a license which requires to sign in.

\section{ACKNOWLEDGEMENTS}

The work has been supported by the Internal CTU grant SGS17/183/OHK3/3T/13 "Special Applications of Signal Processing".

Maple is a trademark of Waterloo Maple Inc.; Maplesoft is a division of Waterloo Maple Inc. All other trademarks are a property of their respective owners.

\section{REFERENCES}

[1] Hurley, William Gerard, and Chi Kwan Lee. "Development, implementation, and assessment of a web-based power electronics laboratory." IEEE Transactions on Education 48.4 (2005): 567573.

[2] J. Bicak, and J. Hospodka. "Symbolic Analysis of Periodically Switched Linear Circuits." SMACD'06-Proceedings of the IX. International Workshop on Symbolic Methods and Applications to Circuit Design. 2006.

[3] J. Hospodka, and J. Bicak. "Web-based application for electric circuit analysis." Computing in the Global Information Technology, 2009. ICCGI'09. Fourth International Multi-Conference on. IEEE, 2009.

[4] Vladimirescu, Andre. The SPICE book. John Wiley \& Sons, Inc., 1994.

[5] Cadence Design Systems, Inc., PSpice - analog and mixedsignal circuit simulator URL: http://www.cadence.com/, 2009.

[6] Spectrum Software, Micro-Cap - schematic editor and mixed analog/digital SPICE circuit simulator URL: http://www.spectrum-soft.com/, 2009.

[7] M. Smith, WinSpice User's Manual http://www.winspice.com, 2007.

[8] Gielen, Georges, Piet Wambacq, and Willy M. Sansen. "Symbolic analysis methods and applications for analog circuits: A tutorial overview." Proceedings of the IEEE 82.2 (1994): 287304.

[9] Beg, Azam. "A web-based method for building and simulating standard cell circuits-A classroom application." Computer Applications in Engineering Education 23.2 (2015): 304-313.

[10] Yang, Ouyang, et al. "ECVlab: A web-based virtual laboratory system for electronic circuit simulation." International Conference on Computational Science. Springer Berlin Heidelberg, 2005 .

[11] Tuma, Tadej, and Árpád Buermen. Circuit simulation with SPICE OPUS: theory and practice. Springer Science \& Business Media, 2009.

[12] Shoufan, Abdulhadi, Zheng Lu, and Sorin A. Huss. "A webbased visualization and animation platform for digital logic design." IEEE Transactions on Learning Technologies 8.2 (2015): 225-239.

[13] Weyten, Ludo, Pieter Rombouts, and Jeroen De Maeyer. "Webbased trainer for electrical circuit analysis." IEEE Transactions on Education 52.1 (2009): 185-189.

[14] Tacha, O. I., et al. "Analysis, adaptive control and circuit simulation of a novel nonlinear finance system." Applied Mathematics and Computation 276 (2016): 200-217.

[15] J. Bicak, and J. Hospodka. "PraCAn-Maple Package for Symbolic Circuit Analysis." Digital Technologies. Z ilina (Slovakia) (2008).

[16] Prusty, Narayan. Learning ECMAScript 6. Packt Publishing Ltd, 2015.

[17] Garrett, Jesse James. "Ajax: A new approach to web applications." (2005). 